\title{
ANÁLISIS DE LA FUNCIÓN Y ULTRAESTRUCTURA MITOCONDRIAL EN RATONES ALBINOS SANOS TRATADOS CON MEDICAMENTOS PARA INSUFICIENCIA CARDÍACA
}

\author{
Analysis of the mitochondrial function and ultrastructure in healthy albino mice treated with heart failure \\ medications
}

\begin{abstract}
Gastón Camino Willhuber ${ }^{1}$, Gustavo Guzman Mentesana'; Alejandra Baez ${ }^{1}$; Silvina Lo Presti ${ }^{1}$; Carolina Bazán ${ }^{1}$; Mariana Strauss ${ }^{1}$; Ricardo Fretes ${ }^{2}$; Patricia Adriana Paglini-Oliva ${ }^{1}$, Hector Walter Rivarola $^{1}$
\end{abstract}

\section{Resumen:}

Introducción: la actividad mitocondrial es esencial para el músculo cardíaco y esquelético. La relación entre la disfunción mitocondrial y diferentes condiciones cardiovasculares ha sido bien descrita. El tratamiento farmacológico de la insuficiencia cardíaca implica diferentes medicamentos como: inhibidores de la enzima convertidora de la angiotensina, bloqueadores B-adrenérgicos, glucósidos digitálicos y diuréticos. Los beneficios clínicos del tratamiento son claros, sin embargo, el papel de estos fármacos en el metabolismo mitocondrial no está bien establecido.

Objetivo del estudio: Analizar las características estructurales y funcionales de las mitocondrias del músculo cardíaco y esquelético en ratones tratados con fármacos habitualmente utilizados para la insuficiencia cardíaca y compararlo con un grupo control.

Métodos: Veinticinco ratones albinos divididos en cinco grupos fueron tratados con la medicación para insuficiencia cardíaca durante 30 días (grupo I a IV). Treinta días después se sacrificaron, el corazón y el músculo esquelético se analizaron y se compararon con un grupo control (V).

Resultados: La actividad enzimática se incrementó ligeramente en los grupos tratados con medicamentos para insuficiencia cardiaca en comparación con el grupo control ( $p>0,05)$. La morfología mitocondrial se modificó significativamente en los grupos tratados en comparación con el grupo control, además, el área mitocondrial fue significativamente mayor en los grupos tratados, tanto en el músculo cardíaco como en el esquelético.

Conclusiones: Concluimos que la medicación para insuficiencia cardíaca podría producir modificaciones en la función mitocondrial; creemos que las mitocondrias pueden mantener la actividad enzimática mediante el aumento de tamaño y modificación de la morfología.

Palabras clave: función mitochondrial; insuficiencia cardiaca; morfología mitocondrial

\section{Abstract:}

Background: Mitochondrial activity is essential for cardiac and skeletal muscle. The relationship between mitochondrial dysfunction and different cardiovascular conditions has been well described. Pharmacological treatment for heart failure involves different drugs as: angiotensin-converting enzyme inhibitors, B-adrenergic blockers, digitalis glycosides and diuretics. The clinical benefit from medication is clear, however, the role of this drugs in mitochondrial metabolisms is not well understood.

Aim of the study: The objective of our study was to analyze structural and functional characteristics of cardiac and skeletal muscle mitochondria in mice treated with drugs normally used for heart failure and compare it to a control group.

Methods: Twenty-five Albino Mice divided in five groups were treated with heart failure medication during 30 days (group I to IV). 30 days after treatment they were sacrificed, heart and skeletal muscle were analyzed and compared with a control group (V).

Results: Enzymatic activity was slightly increased in groups treated with heart failure medication compared to control group ( $p>0.05$ ). Mitochondrial morphology was significantly altered in groups treated compared to control group, in addition, mitochondrial area was significantly increased in the treated groups, in both cardiac and skeletal muscle.

Conclusions: We concluded that heart failure medication could produce modifications in mitochondrial function; we believe that mitochondria maintains the enzymatic activity by increasing size and modifying morphology.

Key words: Mitochondrial function, heart failure medication, Mitochodrial morphology.

\footnotetext{
1. Cátedra de Física Biomédica, Facultad de Ciencias Médicas, Universidad Nacional de Córdoba. Córdoba, Argentina. E-mail: gaston.camino@hiba.org.ar
}

2. Cátedra de Biología Celular, Histología y Embriología, Facultad de Ciencias Médicas, Universidad Nacional de Córdoba. Córdoba, Argentina. 


\section{Introducción}

Las enfermedades cardiovasculares (ECV) son la principal causa de muerte en el mundo ${ }^{(1)}$. Entre las diferentes enfermedades cardiovasculares, la insuficiencia cardíaca crónica (ICC) es un síndrome caracterizado por la incapacidad del corazón para mantener las demandas metabólicas del cuerpo y es responsable del aumento de la morbilidad y la mortalidad ${ }^{(2)}$.

El músculo cardíaco se compone de células estriadas, que se unen a través de las uniones intercelulares que forman los discos intercalados permite la coordinación de la actividad contráctil a modo de sincicio. Las células musculares muestran actividad metabólica importante y un desarrollo significativo de organelas, tales como el retículo endoplasmático liso y rugoso y gran desarrollo de la actividad mitocondrial. Éstas son la principal fuente de energía de las células en nuestro cuerpo, representando el $20-40 \%$ del volumen total de los miocardiocitos y suministrando al corazón un $90 \%$ de la energía necesaria para sus funciones a través de la producción de adenosina trifosfato (ATP) en las proximidades de las miofibrillas, que participan en la actividad contráctil(3).

El número de las mitocondrias está relacionado con la actividad celular. En promedio hay 50-100 mitocondrias en una célula, pero el número puede elevarse considerablemente en función de las necesidades. Incluso la arquitectura tiene importancia, como se muestra por las crestas mitocondriales de la membrana interna de esta organela, cuyo número de pliegues en los miocardiocitos es mucho mayor que en otras células y esto está estrechamente relacionada con la producción de energía. Se ha descrito la relación entre el deterioro de la función mitocondrial a nivel morfológico y funcional y diferentes enfermedades cardiacas tales como: insuficiencia cardíaca ${ }^{(4,5)}$, hipertrofia ${ }^{(6)}$, la isquemia -reperfusión ${ }^{(7,8)}$ etc. Este deterioro podría estar mediado principalmente por la producción de especies reactivas del oxígeno $(\mathrm{ROS})^{(7,9)}$. El manejo de la ICC así como el control de los factores de riesgo es un desafío y uno de los pilares del tratamiento ${ }^{(10)}$.

El tratamiento farmacológico implica distintos grupos de fármacos: diuréticos, los cuales reducen la sobrecarga de volumen; inhibidores de la enzima convertidora de la angiotensina (IECA), que producen vasodilatación, reducen la pre y pos carga y la remodelación cardiaca; bloqueantes beta-adrenérgicos, que reducen la frecuencia cardiaca y disminuyen los requerimientos de oxígeno del miocardio y los glucósidos digitálicos, que aumentan la fuerza de contracción cardiaca (efecto inotrópico positivo) ${ }^{(11,12)}$. Entre los diferentes efectos observados relacionados con los medicamentos se mencionan: aumento o disminución de potasio y de sodio en el plasma, y cambios electrocardiográficos. Estos últimos se presentan con frecuencia con el uso de digoxina y aunque no todos ellos implican la toxicidad, su efecto cardiaco es evidente. Estos cambios nos llevan a considerar los posibles efectos mitocondriales que estos medicamentos pueden producir. Por lo tanto, los objetivos del presente estudio son los siguientes:

Determinar las características estructurales y funcionales de las mitocondrias de las células del músculo cardíaco y esquelético en ratones albinos suizos sanos tratados con: enalapril, espironolactona, atenolol, furosemida, hidroclorotiazida, digoxina y dinitrato de isosorbide, que se utilizan comúnmente en el tratamiento de la insuficiencia cardíaca y compararlo con los ratones no tratados.

\section{Materiales y métodos}

Veinticinco ratones hembras y machos albinos fueron utilizados, divididos en 5 grupos de 5 ratones cada uno, tratados como si presentaran grados progresivos de insuficiencia cardiaca, de acuerdo con la clasificación funcional de la New York Heart Association, que considera el grado 1: aquellos pacientes que sufren de enfermedad cardiaca pero sin limitación de la actividad física, la actividad física frecuente no causa fatiga, palpitaciones, disnea o angina de pecho. Grado 2 : pacientes con enfermedad cardiaca y ligera limitación de la actividad física; asintomáticos en reposo. La actividad física frecuente causa fatiga, disnea, angina de pecho, etc. Grado 3: pacientes con enfermedad cardiaca y limitación significativa de la actividad física; asintomático en reposo. La sintomatología es provocada por actividades menos intensas de lo normal y el grado 4: pacientes con enfermedad cardíaca que permanecen sintomáticos incluso en reposo. Los síntomas son exacerbados por la menor actividad.

Grupo 1: Se consideran insuficiencia cardiaca de grado 1 y en tratamiento con enalapril, espironolactona y atenolol. 
Grupo 2: Se consideran de grado 2 y tratados con enalapril, espironolactona, atenolol e hidroclorotiazida.

Grupo 3: Considerado grado 3 y en tratamiento con enalapril, atenolol y espironolactona, furosemida y digoxina.

Grupo 4: Considerado grado 4 y en tratamiento con enalapril, espironolactona, atenolol, furosemida, digoxina y dinitrato de isosorbida.

Grupo 5: Grupo de control.

Las dosis se calcularon según el peso promedio de los animales (30 $\pm 1 \mathrm{gr}$ ) incluidos en el estudio y fueron por ratón: Enalapril: $2,1 \times 10^{-5} \mathrm{gr}$; Atenolol: $5,3 \times 10^{-5} \mathrm{gr}$; Espironolactona: $1,07 \times 10^{-}$ 4 gr; Furosemida: 4,2 x $10^{-5} \mathrm{gr}$; Hidroclorotiazida; $1,07 \times 10^{-4} \mathrm{gr}$; Digoxina: $6 \times 10^{-6}$ gr y Dinitrato de Isosorbide: $1,07 \times 10^{-5} \mathrm{gr}$. El tratamiento consistió en la administración oral diaria de los medicamentos diluidos en solución fisiológica formando soluciones de $15 \mu \mathrm{l}$ de cada medicación para las cuales se utilizaron micropipetas para la administración de dichas soluciones.

El tratamiento duró 30 días y al cabo de 60 días de iniciado el mismo se procedió al sacrificio de los animales para realizar los análisis correspondientes.

Se llevó a cabo la toma de dos muestras de miocardio a nivel del apex y dos de músculo esquelético de los miembros, para los análisis correspondientes. Con estas muestras se realizó:

\section{Aislamiento mitocondrial}

Las muestras de miocardio de ventrículo izquierdo y una porción de músculo esquelético de aproximadamente $1 \mathrm{~g}$ fueron lavados $\mathrm{y}$ suspendidos en un buffer de aislamiento helado (5 mM HEPES, pH 7.2 containing $210 \mathrm{mM}$ mannitol, $70 \mathrm{mM}$ sucrose, $1 \mathrm{mM}$ EGTA and $0.5 \%$ BSA, libre de ácidos grasos, 1:10 p/v) e inmediatamente homogeneizados. Los homogenatos se centrifugaron a $1000 \mathrm{~g}, 4^{\circ} \mathrm{C}$ por 5 min y el sobrenadante fue transferido a un nuevo tubo. El pellet se suspendió en el buffer de aislamiento, se homogeneizó y se centrifugó nuevamente como antes. Los sobrenadantes se combinaron y centrifugaron a $8100 \mathrm{~g}$ por $15 \mathrm{~m}$ a $4^{\circ} \mathrm{C}$. El pellet rico en mitocondrias se resuspendió en buffer de aislamiento (relación tejido/buffer: 1/1) y las alícuotas se conservaran a $-80^{\circ} \mathrm{C}$. La concentración de proteínas se midió por el método de Lowry ${ }^{(16)}$.

Esta fracción sirvió para determinar:

Actividad del complejo III de la cadena respiratoria
La cuantificación de la actividad enzimática mitocondrial se realizó a través del control de la actividad de la: ubiquinina-citocromo c-reductasa (complejo III) por espectrofotometría ${ }^{(17,18,19)}$ Los resultados se expresaron en $\mu \mathrm{mol}$ de Citocromo $C$ por minuto por miligramo de proteinas.

Análisis morfológico mitocondrial: Se realizaron cortes finos y se contrastaron con acetato de uranilo y citrato de plomo a los fines de ser analizados en microscopio electrónico con magnificación de 1000 y $27800 \mathrm{X}$.

Se realizó un análisis de la morfología mitocondrial de muestras obtenidas de miocardio ventricular y músculo esquelético. Se consideró normal (ver figura 1.A) a aquellas mitocondrias que no exhibieron alteraciones en el tamaño ni en la forma y cuyas crestas mitocondriales presentaban un ordenamiento armónico, sin distorsión. Se consideró Grado 1 (ver figura 1.B) a aquellas mitocondrias que mostraron forma y tamaño irregular, distorsión de las crestas mitocondriales, ruptura de crestas (crestólisis), aumento de la matriz. Se consideró Grado 2 (ver figura 1.C) a la presencia de disrupción de la membrana mitocondrial externa, como expresión de daño mitocondrial irreversible.

Análisis estadístico: De acuerdo a la naturaleza de la variable (ANOVA y Test de Fisher para variables cuantitativas (actividad enzimática) y Chi cuadrado para variables cualitativas (morfología mitocondrial) este último a través de tablas de contingencia. La cuantificación mitocondrial mediante el programa Axiovisión 3.0, estableciéndose diferencias significativas cuando $\mathrm{p}<0.05$ para todos los casos.

\section{Resultados}

No se observaron alteraciones en las mitocondrias de los ratones del grupo 5, sin tratamiento, (Fig. $1 \mathrm{~A})$. En el miocardio ventricular de ratones tratados pertenecientes al grupo 1 se detectaron alteraciones en las mitocondrias como: crestólisis, dilatación de crestas y aumento de matriz (Fig. $1 \mathrm{~B}$ ). En los ratones tratados del grupo 2 se detecto disrupción de membrana mitocondrial externa (Fig. $1 \mathrm{C}$ ) 


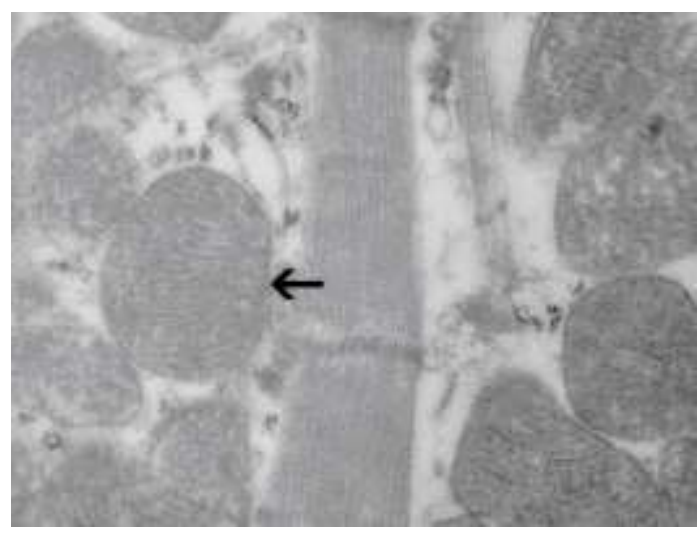

A
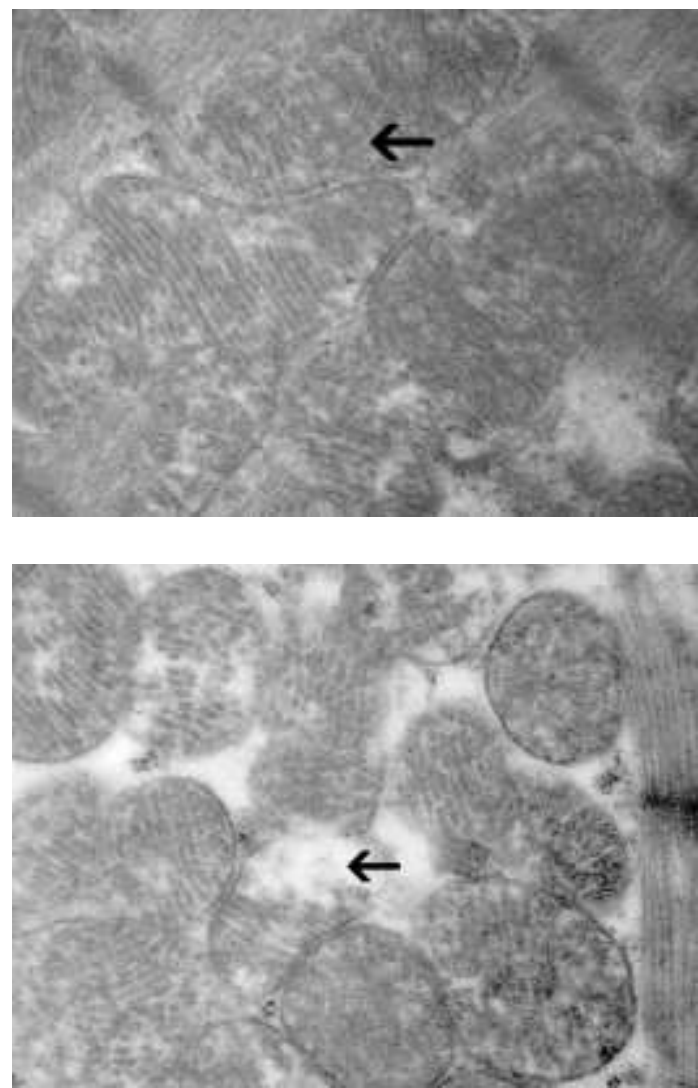

Figura 1. A: Mitocondrias de miocardio ventricular obtenidas de ratones sin tratamiento (Normal) B: Mitocondrias de miocardio ventricular obtenida de ratones tratados, se observa morfología alterada (crestolisis, dilatación de crestas, aumento de matriz) (Grado 1). C: Mitocondrias obtenidas de miocardio ventricular en ratones tratados, se observa disrupción de membrana mitocondrial externa (Grado 2).

En la tabla 1 se observan los porcentajes de mitocondrias normales (Normal) de alteraciones leves y moderadas (Grado1) y de alteraciones severas (Grado 2). El análisis estadístico arrojó diferencias significativas entre el control y los grupos tratados $(p<0,05)$. En los grupos tratados disminuye significativamente el porcentaje de mitocondrias sin alteraciones y aumenta el porcentaje de alteraciones morfológicas con respecto al control en todos los grupos tratados. Es de destacar que el grupo 1 y el grupo 2 mostraron diferencias significativas $(p<0,05)$ de mitocondrias sin alteraciones. 
Tabla 1. Porcentaje de modificaciones morfológicas mitocondriales en músculo cardíaco, GI a GIV (grupos tratados) $n=25$.

\begin{tabular}{|c|c|c|c|c|c|}
\hline & CONTROL & GI & GII & GIII & GIV \\
\hline NORMAL & $50,81 \%$ & $22,58 \%$ & $28,26 \%$ & $7,07 \%$ & $4,12 \%$ \\
\hline GRADO 1 & $45,9 \%$ & $64,51 \%$ & $60 \%$ & $64,3 \%$ & $79,12 \%$ \\
\hline GRADO 2 & $3,27 \%$ & $12,9 \%$ & $11,75 \%$ & $28,51 \%$ & $16,62 \%$ \\
\hline
\end{tabular}

Actividad enzimática en músculo cardíaco: El análisis de la actividad enzimática mitocondrial del músculo cardíaco, estudiada a través de la actividad del complejo III de la cadena respiratoria mostró un aumento significativo en el grupo 1 con respecto al grupo control no observándose diferencias con respecto a los demás grupos tratados. (Ver figura 2).

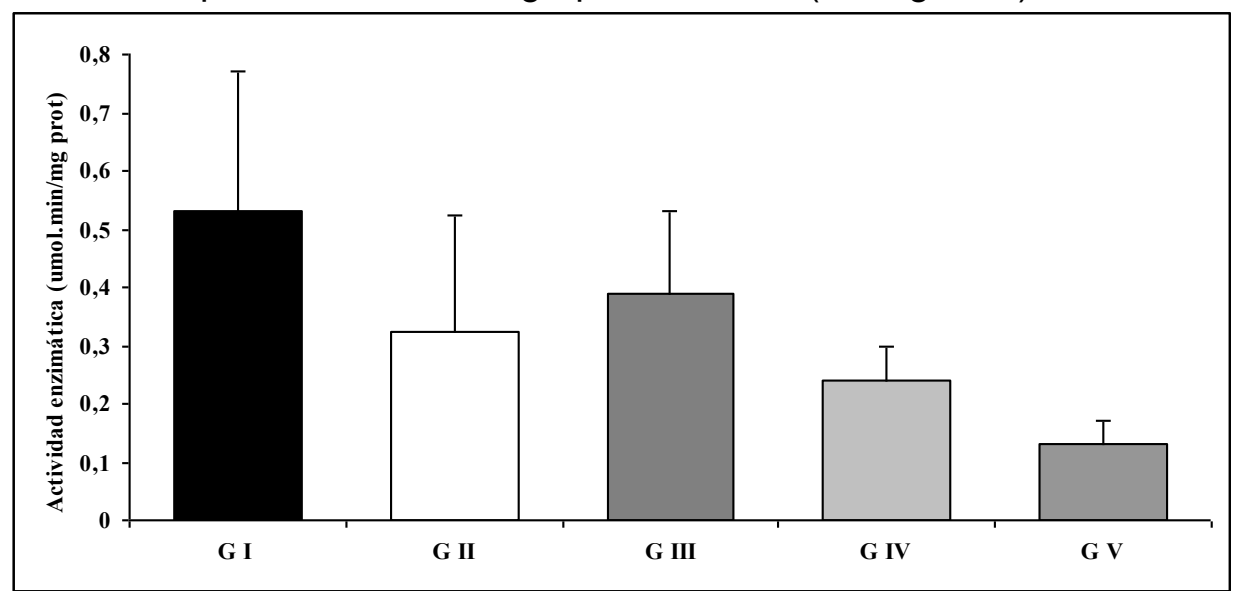

Figura 2. Actividad enzimática del complejo III de la cadena respiratoria mitocondrial del músculo cardíaco en grupos tratados (GI a GIV) y V (control). Expresada en $\mu$ mol de Citocromo $\mathrm{C} /$ minuto/ miligramos de Proteinas. $\mathrm{n}=25$

Área mitocondrial en músculo cardíaco: Como se puede observar en la figura 3 existe un aumento significativo $(p<0,05)$ de la superficie mitocondrial de los grupos 3 y 4 con respecto al control, los grupos 1 y 2 no arrojan diferencias significativas con respecto al grupo control.

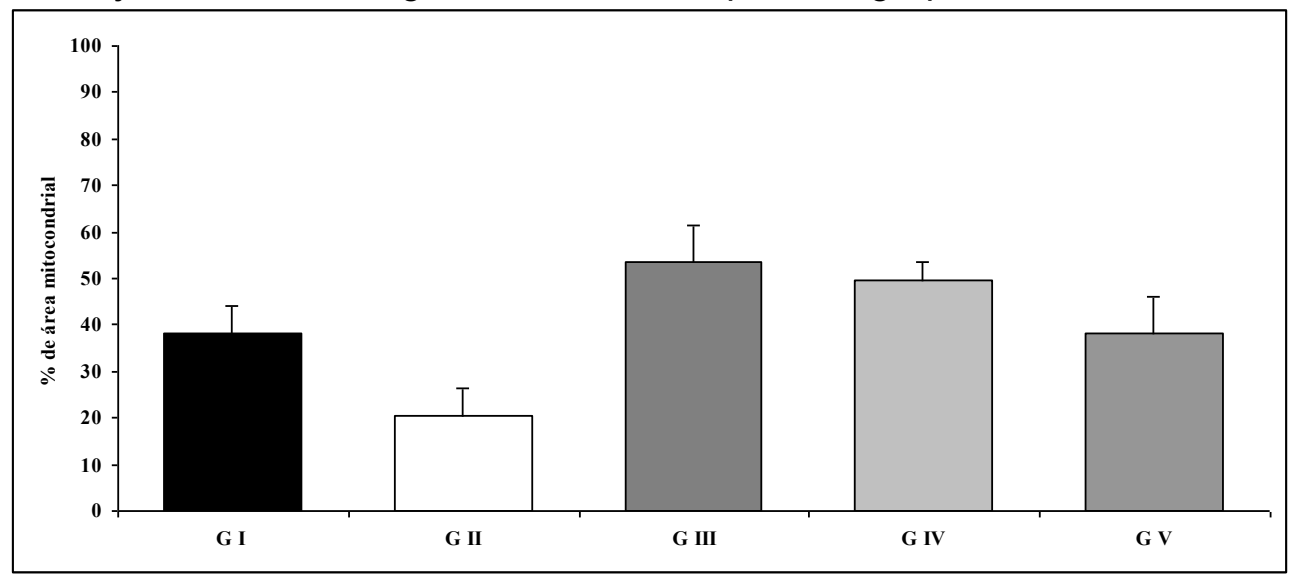

Figura 3. Porcentaje de área mitocondrial en músculo cardíaco en grupos tratados (GI a GIV) y $\vee$ (grupo control). $n=25$.

\section{Músculo esquelético}

\section{Análisis morfológico mitocondrial}

Se observaron diferencias significativas entre el grupo control y los grupos tratados $(p<0,05)$ los cuales presentan mayor proporción de alteraciones grado 1 y 2 y menor porcentaje de mitocondrias sin alteraciones. Nuevamente se destaca que los grupos 1 y 2 muestran un mayor porcentaje de mitocondrias sin alteraciones $(p<0,05)($ Ver tabla 2$)$ 
Tabla 2. Porcentaje de modificaciones morfológicas mitocondriales en músculo esquelético, GI a G IV (grupos tratados) y G V (control).

\begin{tabular}{|l|l|l|l|l|l|}
\hline & $\begin{array}{c}\text { CONTROL } \\
(G)\end{array}$ & GI & GII & GIII & GIV \\
\hline NORMAL & $83,33 \%$ & $62,47 \%$ & $66,75 \%$ & $39,49 \%$ & $9,01 \%$ \\
\hline GRADO 1 & $16,66 \%$ & $37,52 \%$ & $33,25 \%$ & $57,89 \%$ & $63,66 \%$ \\
\hline GRADO 2 & $0 \%$ & $0 \%$ & $0 \%$ & $2,6 \%$ & $27,32 \%$ \\
\hline
\end{tabular}

Actividad enzimática en músculo esquelético: El análisis de la actividad enzimática del completo III de la cadena respiratoria no mostró diferencias significativas. Los resultados son similares a los observados en músculo cardíaco. (Ver figura 4).

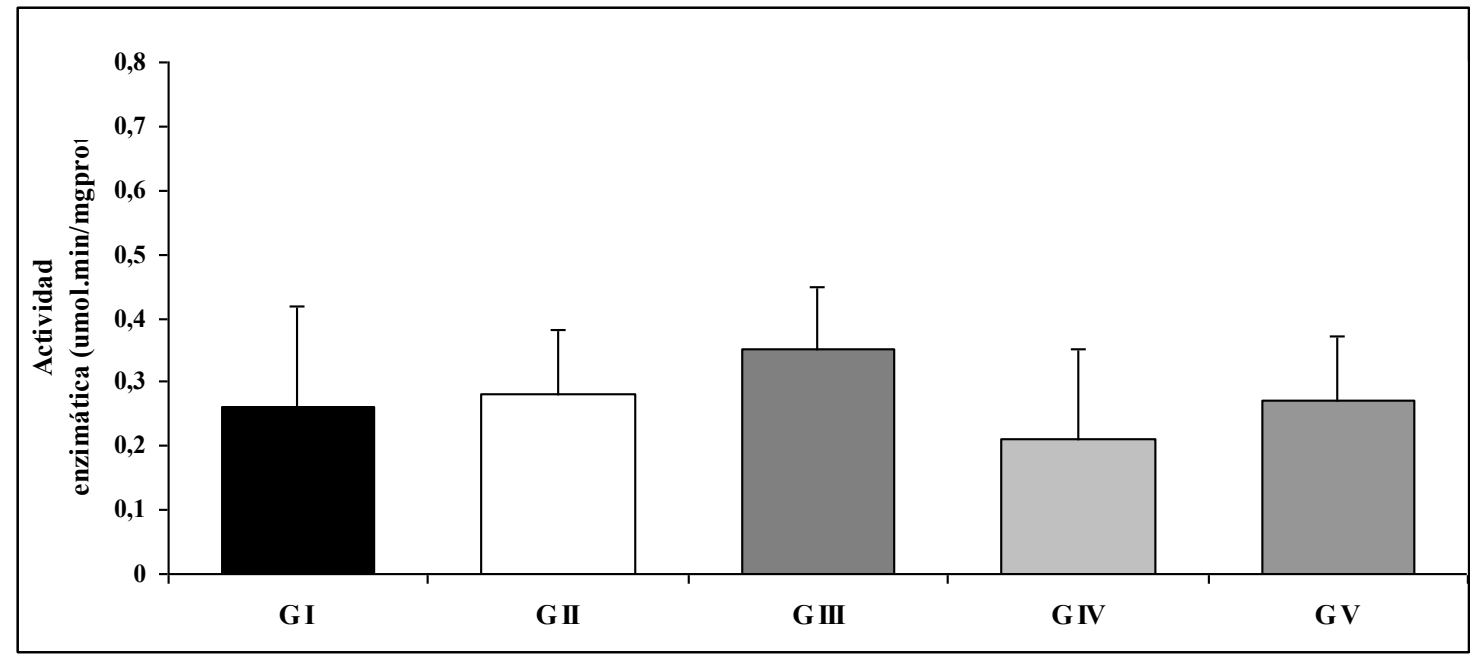

Figura 4. Actividad enzimática mitocondrial del músculo esquelético en grupos tratados (GI a GIV) y $V$ (control). Complejo III de la cadena respiratoria. Expresada en $\mu \mathrm{mol}$ de Citocromo $\mathrm{C} /$ minuto/ miligramos de Proteínas. $\mathrm{n}=25$.

Área mitocondrial en músculo esquelético: Como se puede observar en la Figura 5 el área mitocondrial está significativamente aumentada en los Grupos III y IV con respecto a los grupos I, II $y$ al grupo control $(V)(p<0,05)$.

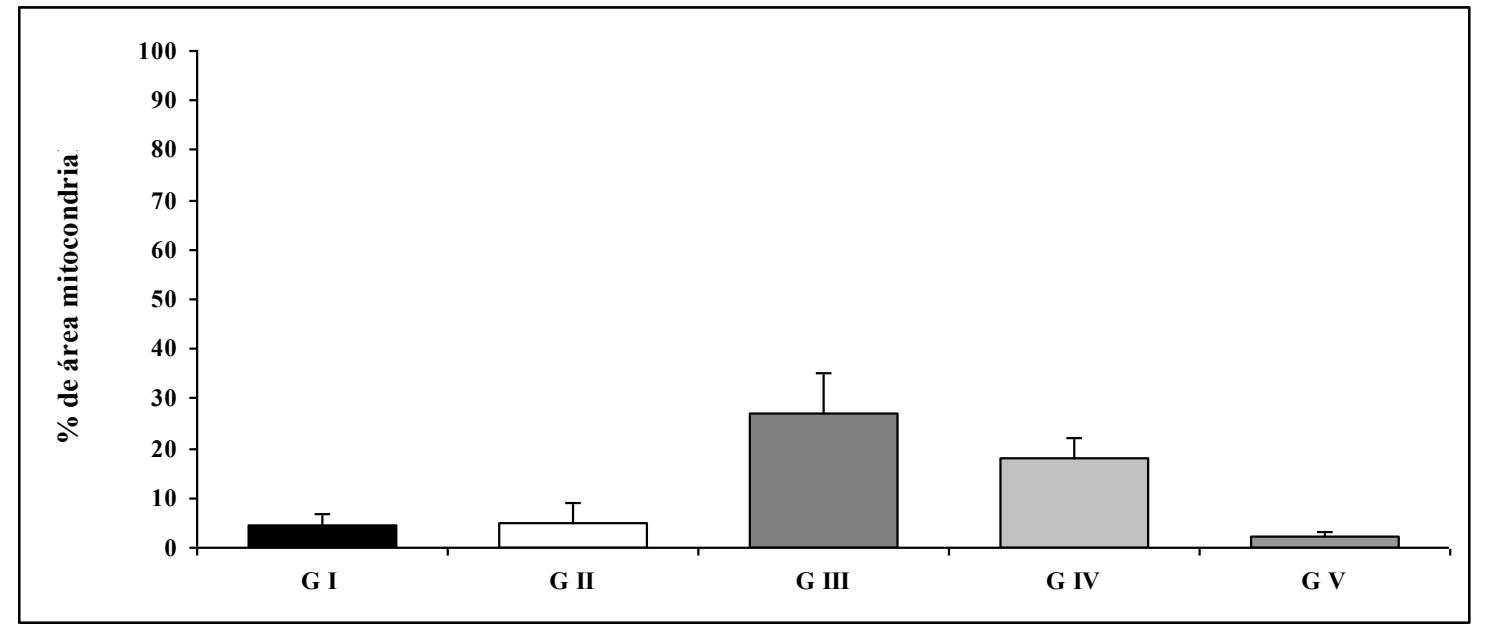

Figura 5. Porcentaje de área mitocondrial en músculo esquelético de grupos tratados (GI a GIV) y $\vee$ (control). $n=25$. 


\section{Electrocardiograma}

Se observó una disminución significativa $(p<0,05)$ de la frecuencia cardíaca en el $G$ II $(283,33 \pm 10$ latidos/minuto) con respecto a los demás grupos (450 \pm 15 latidos/minuto), no se observaron otra modificaciones electrocardiográficas.

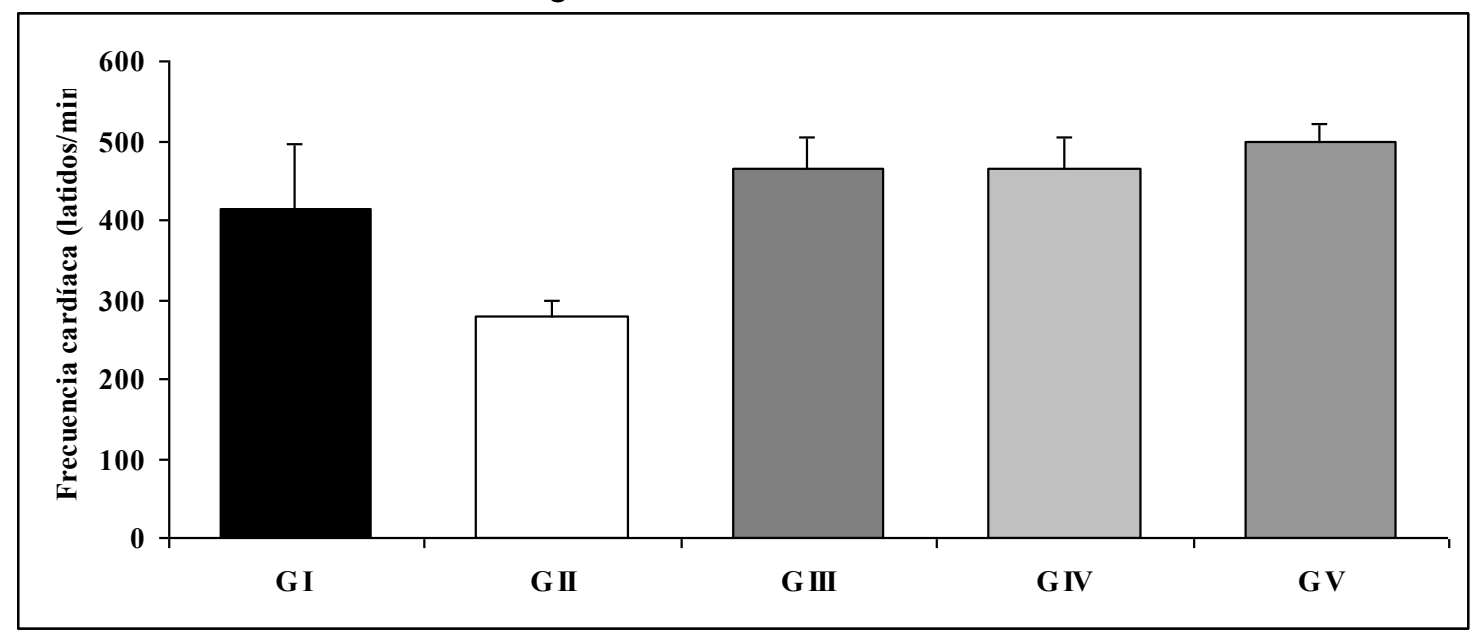

Figura 6. Frecuencia cardíaca (latidos $/ \mathrm{min}$ ) de grupos tratados I a IV y $V$ (controles). $n=25$

\section{Discusión}

La actividad del musculo cardíaco, necesaria para cumplir su función, requiere un importante desarrollo mitocondrial. Las mitocondrias son abundantes en el corazón, donde representan el $20-40 \%$ del volumen celular, debido a la alta demanda energética ${ }^{(3)}$.

El conocimiento de la relación entre la disfunción mitocondrial y la fisiopatología de diversas enfermedades $^{(18)}$, tales como neurodegenerativas [19] y las enfermedades cardiovasculares ${ }^{(20,21)}$ es amplia y permite apreciar el papel significativo que desempeñan estas organelas en el proceso de salud-enfermedad.

En cuanto a la relación entre los fármacos utilizados para patologías cardiovasculares y modificaciones funcionales y morfológicas mitocondriales, los resultados varían, y algunos muestran efectos claramente de protección, tales como inhibidores de la enzima convertidora de angiotensina(IECA) a nivel $\operatorname{cardiac}^{(23,26)}$. Sin embargo, se mostró que estos efectos en modelos de hipertensión e hipertrofia miocárdica y no en los corazones normales. Se han observado cambios mitocondriales inducidos por fármacos en estudios anteriores ${ }^{(27,28)}$. Además, los resultados anteriores en nuestra institución mostraron daño mitocondrial mediado por especies reactivas de oxígeno en pacientes con enfermedad cardiaca crónica en comparación con un grupo control ${ }^{(22)}$.

En el presente estudio, no se observaron diferencias significativas funcionales entre los grupos tratados con medicamentos comúnmente utilizados para la insuficiencia cardíaca y el grupo control, la actividad de la función mitocondrial, analizada a través del complejo III de la cadena respiratoria, no mostró diferencias significativas en los grupos tratados en comparación con el grupo control, ya sea en el músculo cardíaco o esquelético. Estos resultados son similares a los observados con el uso de digoxina ${ }^{(26)}$, a diferencia de otros estudios, que muestran una disminución en la actividad de enzimas cardíacas con el uso de bloqueadores beta ${ }^{(29)}$. Otro estudio mostró que el uso de diuréticos (furosemida, hidroclorotiazida) redujo la actividad mitocondrial a nivel renal ${ }^{(30)}$. No hemos encontrado estudios que analizan el efecto de los diuréticos a nivel cardíaco y esquelético.

Estos resultados permiten inferir que la actividad de la enzima se ha modificado ligeramente.

El presente estudio muestra un aumento en el porcentaje de modificaciones estructurales (cristolysis, sus crestas dilatada, la matriz ampliada, degeneración hidrópica) en las mitocondrias en comparación con los grupos tratados con respecto al grupo de control tanto en los músculos cardíaco y esquelético. Este aumento fue mayor en los grupos III y IV, que recibieron más medicamentos. Estos resultados son similares a los observados en otros estudios, que muestran el daño celular y el aumento de la apoptosis mediada por altas dosis de digoxina ${ }^{(31)}$ y el daño ultra estructural mediado por nitroglicerina (cuyo 
mecanismo de acción es similar al dinitrato de isosorbida) relacionado con el aumento de la producción de $\mathrm{ROS}^{(32)}$. Estos resultados podrían explicar en parte la mayor proporción de modificaciones estructurales en las mitocondrias en los grupos III y IV.

Se observó un aumento significativo de la superficie mitocondrial $(p<0,05)$ en los tejidos cardiacos y esqueléticos de los grupos tratados con más medicamentos (III y IV). Este fenómeno asociado al mayor porcentaje de alteraciones mitocondriales podría explicar que el mantenimiento de la actividad enzimática mitocondrial se produce a expensas de un aumento de su área y el mayor porcentaje de alteraciones de la morfología mitocondrial en estos grupos se compensado por un aumento de la zona con el fin para mantener la función mitocondrial en comparación con el grupo control.

\section{Conclusión}

Estos resultados permiten inferir que la función mitocondrial se mantiene a expensas de un aumento del tamaño, lo que tendría relación con las modificaciones estructurales compatibles con daño mitocondrial tanto en los músculos cardíacos y esqueléticos.

Esto además, es una contribución al conocimiento del comportamiento de estos medicamentos en los niveles de función ultra estructural y mitocondrial, lo que conduce a nuevas preguntas con respecto a la función mitocondrial complejo.

\section{Bibliografía}

1- Organización Mundial de la Salud. Sedentary lifestyle: A

Global Public Health Problem. Ginebra: OMS; 2002.

2- Francis GS. Pathophysiology of the heart failure clinical syndrome. EL Topol, ed. Textbook ok cardiovascular medicine. Filadelfia, Lippincott-Raven Publishers, 1997.

3- Marin-García J. ; GoldenthalMJ. Mitochondria and the heart. Rev Esp Cardiol 2002; 55:1293-1310.

4- Marin-Garcia J. Mitochondrial dysfunction in heart failure. J Am Coll Cardiol 2002; 40:2174-2181.

5- Marin-Garcia J, Goldenthal MJ, Pierpont ME, et al. Impaired mitochondrial function in idiopathic dilated cardiomyopathy: Biochemical and Molecular análisis. J Card Fail 1995:285-291.

6- Izgi C, Cevik C, Bakal RB, et al. Severe obstructive hypertrophic cardiomyopathy occurring secondary to mitochondrial disease. Turk Kardiyol Dern Ars. 2009; 37:332-336. 7- Gao L, Laude K, Cai H. Mitochondrial pathophysiology, reactive oxygen species, and cardiovascular diseases. Vet Clin North Am Small Anim Pract 2008; 38:137-155.

8- Tompkins AJ, Burwell LS, Digerness SB, et al. Mitochondrial dysfunction in cardiac ischemia-reperfusion injury: ROS from complex I, without inhibition. Biochim Biophys Acta 2006:223-231.

9- Tsutsi $H$. Oxidative stress in heart failure : the role of mitochondria. Int. Med 2006; 40, 1177- 1182.

10- Ho KK, Pinsky JL, Kannel WB, et al. The epidemiology of heart failure: the Framingham Study. J Am Coll Cardiol 1993; 22:6-13.

11- Fernando de la Serna. Tratamiento de insuficiencia cardíaca. Actualidad y perspectivas.Rev Fed Arg Cardiol 1999; 28: $217-$ 228.

12- Smith TW, Kelly RA, LW Stevenson, et al. The management of heart failure. En: Braunwald E, ed. Heart disease, 5ta ed. Filadelfia, WB Saunders, 1994.

13- Hurst JW, Morris DC, Alexander RW. The use of the New York Heart Association's classification of cardiovascular disease as part of the patient's complete Problem List. Clin Cardiol 1999; 22:385-390.

14- Oliver H, Lowry Nira J, Rosebrough A, et al. Protein measurement with the folin phenol reagent. J. Biol. Chem 1951;193: 265-275.

15- Rickwood, D.; Wilson, M.T.; Darley-Usmar, V.M. En Mitocohondria: A practical approach (Darley-Usmar, Rickwood, Wilson, eds.) pp 1-16. Oxford, IRL Press Limited. 1987.

16- Trounce IA, Kim YL, Jun AS, et al. Assessment of mitochondrial oxidative phosphorylation in patient muscle biopsies, lymphoblasts, and transmitochondrial cell lines, Methods Enzymol 1996;264: 484-509.

17- Jarreta $D$, Orus $J$, Barrientos $A$, et al.Mitochondrial function in heart muscle from patients with idiopathic dilated cardiomyopathy, Cardivasc. Res 2000; 45: 860-865.

18- Seppet E, Gruno M, Peetsalu A, et al. Mitochondria and energetic depression in cell pathophysiology. Int J Mol Sci 2009; 19:2252-2303.

19- Su B, Wang $X$, Zheng $L$, et al. Abnormal mitochondrial dynamics and neurodegenerative diseases. Biochim Biophys Acta 2009;30.

20- Nojiri H, Shimizu T, Funakoshi $M$, et al. Oxidative stress causes heart failure with impaired mitochondrial respiration. J Biol Chem. Nov 2006; 3;281:3789-3801.

21- Marín-García J, Goldenthal MJ. Mitochondrial centrality in heart failure. Heart Fail Rev 2008;13:137-150.

22- Guzman Mentesana G, Baez A, Córdoba $R, L$ L. Role of mitochondria and reactive oxygen species in the progression of heart failure. Rev Fac Cien Med Univ Nac Cordoba 2010;67:150158.

23- Zahorodnyı̆ MI, Stechenko LO, Kuftyrieva TP, et al. Influence of lipril on the myocardial ultrastructure in rats with spontaneous hypertension. Lik Sprava. 2008:82-87.

24- de Cavanagh EM, Piotrkowski B, Basso N, et al. Enalapril and losartan attenuate mitochondrial dysfunction in aged rats. FASEB j 2003;9:1096-1098.

25- de Cavanagh EM, Piotrkowski B, Fraga CG. Concerted action of the renin-angiotensin system, mitochondria, and antioxidant defenses in aging.Mol Aspects Med 2004; 25:27-36.

26- Tsyganii AA, Medvinskaia NA, Rudenko AF. Effect of digoxin, strophanthin and isolanid on oxygen absorption, oxidative 
phosphorylation and the amount of cytochromes in the myocardial mitochondria and their ATPase activity. Farmakol Toksikol 1982;45:30-32.

27- Pereira GC, Silva AM, Diogo CV, et al. Drug-induced cardiac mitochondrial toxicity and protection: from doxorubicin to carvedilol. Curr Pharm Des 2011;17:2113-2129.

28- Syed M, Skonberg C, Hansen SH. Mitochondrial toxicity of diclofenac and its metabolites via inhibition of oxidative phosphorylation (ATP synthesis) in rat liver mitochondria: Possible role in drug induced liver injury (DILI). Toxicol In Vitro 2016; 31:93-102.

29-Quinn PJ, Crutcher EC. The action of beta-adrenoceptor antagonists on rat heart mitochondrial function in vitro: a comparison of propranolol, timolol, and atenolol. Cardiovasc Res 1984;18:212-219.

30- Eknoyan G, Sawa H, Hyde S, et al. Effect of diuretics on oxidative phosphorylation of dog kidney mitochondria.J Pharmacol Exp Ther 1975;194:614-623.

31- Ramirez-Ortega M, Zarco G, Maldonado V, et al. Is digitalis compound-induced cardiotoxicity, mediated through guinea-pig cardiomyocytes apoptosis?. Eur J Pharmacol 2007;566:1-3:3442.

32- Gori T, Daiber A, Di Stolfo G, Sicuro S, et al. Nitroglycerine causes mitochondrial reactive oxygen species production: in vitro mechanistic insights. Can J Cardiol 2007; 23:990-992. 